\title{
Colliding waves in metric-affine gravity
}

\author{
Alberto García ${ }^{a *}$, Claus Lämmerzahl ${ }^{b \dagger}$, \\ Alfredo Macías ${ }^{c \ddagger}$, Eckehard W. Mielke ${ }^{c \S}$, and José Socorro ${ }^{d * *}$ \\ ${ }^{a}$ Departamento de Física, \\ CINVESTAV-IPN, Apartado Postal 14-740, C.P. 07000, México, D.F., MEXICO \\ ${ }^{b}$ Fakultät für Physik, Universität Konstanz \\ Postfach 5560 M674, D-78434 Konstanz, Germany \\ ${ }^{c}$ Departamento de Física, \\ Universidad Autónoma Metropolitana-Iztapalapa, \\ Apartado Postal 55-534, C.P. 09340, México, D.F., MEXICO. \\ ${ }^{d}$ Instituto de Física de la Universidad de Guanajuato, \\ Apartado Postal E-143, C.P. 37150, León, Guanajuato, MEXICO.
}

(September 25, 2018)

\begin{abstract}
We generalize the formulation of the colliding gravitational waves to metricaffine theories and present an example of such kind of exact solutions. The plane waves are equipped with five symmetries and the resulting geometry after the collision possesses two spacelike Killing vectors.
\end{abstract}

\footnotetext{
*E-mail: aagarcia@fis.cinvestav.mx

†E-mail: claus@spock.physik.uni-konstanz.de

†E-mail: amac@xanum.uam.mx

§E-mail: ekke@xanum.uam.mx

**E-mail: socorro@ifug4.ugto.mx
} 
PACS numbers: 04.50.+h; 04.20.Jb; 03.50.Kk

Typeset using REVTEX 


\section{INTRODUCTION}

In recent years, the collision of plane-fronted gravitational waves possibly coupled with electromagnetic waves, has been extensively studied [1] [4]. Because gravity is always attractive, it was expected that focusing of the waves would occur and one of the interesting questions is how much focusing does general relativity predict. Within this framework, strong focusing would appear by the development of spacetime curvature singularities. Many solutions has been presented so far, describing the collisions of plane-fronted gravitational and electromagnetic waves. And quite a few of them do develop Cauchy horizons.

The spacetimes describing the interaction region produced after the collision of plane gravitational waves contain two spacelike Killing vectors and there exist several generating techniques to obtain solutions with these symmetries. All the techniques developed for stationary axysimmetric spacetimes can be applied to generate cylindrically symmetric spacetimes, in particular, colliding plane waves.

On the other hand, if one gauges the affine group and additionally allows for a metric $g$, then one ends up with the metric-affine gauge theory of gravity (MAG) [5]. The fourdimensional affine group $A(4, \mathbf{R})$ is the semidirect product of the translation group $R^{4}$ and the linear group $G L(4, \mathbf{R})=R^{+} \otimes[T \otimes S L(4, \mathbf{R})]$. This spacetime encompasses two different post-Riemannian structures: the nonmetricity one-form $Q_{\alpha \beta}=Q_{i \alpha \beta} d x^{i}$ and the torsion two-form $T^{\alpha}=\frac{1}{2} T_{i j}{ }^{\alpha} d x^{i} \wedge d x^{j}$. In the Yang-Mills fashion, gauge Lagrangians quadratic in curvature, torsion, and nonmetricity are considered. One way to investigate the potentialities of such models is to look for exact solutions.

The search for exact solutions within MAG has been pioneered by Tresguerres [6,7] and by Tucker and Wang [8]. With propagating nonmetricity $Q_{\alpha \beta}$, two types of charge are expected to arise: One dilation charge related by Noether procedure to the trace $Q:=Q_{\gamma}^{\gamma} / 4$ of the nonmetricity, called the Weyl covector $Q=Q_{i} d x^{i}$. It is the connection associated with gauging $\mathbf{R}^{+}$instead of $U(1)$ for the Maxwell potential $A=A_{i} d x^{i}$. Nine types of shear charge related to the remaining traceless piece $\varnothing_{\alpha \beta}:=Q_{\alpha \beta}-Q g_{\alpha \beta}$ of the nonmetricity. 
Under the local Lorentz group, the nonmetricity can be decomposed into four irreducible pieces ${ }^{(I)} Q_{\alpha \beta}$, with $I=1,2,3,4$. The Weyl covector is linked to ${ }^{(4)} Q_{\alpha \beta}=Q g_{\alpha \beta}$.

The following natural step in these lines is to elucidate the behavior of interacting plane waves. Of particular relevance is the head-on collision of two plane waves, i.e. the colliding wave problem. It is assumed that in the corresponding spacetime, the two waves approach each other, from opposite sides, in flat Minkowski background; after the collision, a new gravitational field evolves, which satisfies certain continuity conditions. The plane waves are equipped with five symmetries, while the geometry resulting after the collision possesses two spacelike Killing vectors. The main pruporse of this paper is to generalize the formulation of the colliding waves to MAG theories and to present an example of such kind of solutions. We will take advantage of the fact that certain MAG models can be reduced to an effective Einstein-Proca system [9]. Macías et al. [10], and Socorro et al. [11] mapped the EinsteinMaxwell sector of the dilaton-gravity coming from low energy string theory, to MAG, thus, finding soliton and multipole solutions.

The plan of the paper is as follows: In Sec. 2 the quadratic MAG Lagrangian is revisited. In Sec. 3 the generalization of the colliding waves concept to MAG is developed. In Sec. 4 a colliding wave solution in MAG is presented. In Sec. 5 the results are discussed.

\section{QUADRATIC MAG LAGRANGIAN}

In a metric-affine spacetime, the curvature has eleven irreducible pieces, see [5], Table 4. If in addition we recall that the nonmetricity has four and the torsion three irreducible pieces, then a general quadratic Lagrangian in MAG reads:

$$
\begin{aligned}
V_{\mathrm{MAG}} & =\frac{1}{2 \kappa}\left[-a_{0} R^{\alpha \beta} \wedge \eta_{\alpha \beta}-2 \lambda \eta+T^{\alpha} \wedge^{*}\left(\sum_{I=1}^{3} a_{I}{ }^{(I)} T_{\alpha}\right)\right. \\
& \left.+2\left(\sum_{I=2}^{4} c_{I}{ }^{(I)} Q_{\alpha \beta}\right) \wedge \vartheta^{\alpha} \wedge^{*} T^{\beta}+Q_{\alpha \beta} \wedge^{*}\left(\sum_{I=1}^{4} b_{I}{ }^{(I)} Q^{\alpha \beta}\right)\right] \\
& -\frac{1}{2} R^{\alpha \beta} \wedge^{*}\left(\sum_{I=1}^{6} w_{I}{ }^{(I)} W_{\alpha \beta}+\sum_{I=1}^{5} z_{I}{ }^{(I)} Z_{\alpha \beta}\right) .
\end{aligned}
$$


In the above, the Minkowsi metric is $o_{\alpha \beta}=\operatorname{diag}(-+++), \eta:={ }^{*} 1$ is the volume four-form and the constants $a_{0}, \cdots a_{3}, b_{1}, \cdots b_{4}, c_{2}, c_{3}, c_{4}, w_{1}, \cdots w_{6}, z_{1}, \cdots z_{5}$ are dimensionless. In the curvature square term we have introduced the irreducible pieces of the antisymmetric part $W_{\alpha \beta}:=R_{[\alpha \beta]}$ and the symmetric part $Z_{\alpha \beta}:=R_{(\alpha \beta)}$ of the curvature two-form. Again, in $Z_{\alpha \beta}$, we meet a purely post-Riemannian part. The segmental curvature ${ }^{(4)} Z_{\alpha \beta}:=R_{\gamma}^{\gamma} g_{\alpha \beta} / 4=$ $g_{\alpha \beta} d Q$ has formally a similar structure as the electromagnetic field strength $F=d A$.

Let us recall the three general field equations of MAG, see [5] Eqs.(5.5.3)-(5.5.5). Because of its redundancy, we omit the zeroth field equation with its gauge momentum $M^{\alpha \beta}$. The first and the second field equations read

$$
\begin{gathered}
D H_{\alpha}-E_{\alpha}=\Sigma_{\alpha}, \\
D H_{\beta}^{\alpha}-E_{\beta}^{\alpha}=\Delta_{\beta}^{\alpha},
\end{gathered}
$$

where $\Sigma_{\alpha}$ and $\Delta^{\alpha}{ }_{\beta}$ are the canonical energy-momentum and hypermomentum current threeforms associated with matter. We will consider the vacuum case with $\Sigma_{\alpha}=\Delta^{\alpha}{ }_{\beta}=0$. The left hand sides of (2.2)-(2.3) involve the gravitational gauge field momenta two-forms $H_{\alpha}$ and $H_{\beta}^{\alpha}$ (gravitational "excitations"). We find them, together with $M^{\alpha \beta}$, by partial differentiation of the Lagrangian (2.1):

$$
\begin{aligned}
M^{\alpha \beta}:= & -2 \frac{\partial V_{\mathrm{MAG}}}{\partial Q_{\alpha \beta}}=-\frac{2}{\kappa}\left[*\left(\sum_{I=1}^{4} b_{I}^{(I)} Q^{\alpha \beta}\right)\right. \\
& \left.+c_{2} \vartheta^{(\alpha} \wedge^{*(1)} T^{\beta)}+c_{3} \vartheta^{(\alpha} \wedge^{*(2)} T^{\beta)}+\frac{1}{4}\left(c_{3}-c_{4}\right) g^{\alpha \beta *} T\right], \\
H_{\alpha}:= & -\frac{\partial V_{\mathrm{MAG}}}{\partial T^{\alpha}}=-\frac{1}{\kappa} *\left[\left(\sum_{I=1}^{3} a_{I}{ }^{(I)} T_{\alpha}\right)+\left(\sum_{I=2}^{4} c_{I}{ }^{(I)} Q_{\alpha \beta} \wedge \vartheta^{\beta}\right)\right], \\
H^{\alpha}{ }_{\beta}:= & -\frac{\partial V_{\mathrm{MAG}}}{\partial R_{\alpha}{ }^{\beta}}=\frac{a_{0}}{2 \kappa} \eta^{\alpha}{ }_{\beta}+\mathcal{W}^{\alpha}{ }_{\beta}+\mathcal{Z}^{\alpha}{ }_{\beta},
\end{aligned}
$$

where we introduced the abbreviations

$$
\mathcal{W}_{\alpha \beta}:=*\left(\sum_{I=1}^{6} w_{I}^{(I)} W_{\alpha \beta}\right), \quad \mathcal{Z}_{\alpha \beta}:=*\left(\sum_{I=1}^{5} z_{I}^{(I)} Z_{\alpha \beta}\right) .
$$

Finally, the three-forms $E_{\alpha}$ and $E^{\alpha}{ }_{\beta}$ describe the canonical energy-momentum and hypermomentum currents of the gauge fields themselves. One can write them as follows [5]: 


$$
\begin{aligned}
E_{\alpha} & \left.\left.\left.\left.=e_{\alpha}\right\rfloor V_{\mathrm{MAG}}+\left(e_{\alpha}\right\rfloor T^{\beta}\right) \wedge H_{\beta}+\left(e_{\alpha}\right\rfloor R_{\beta}{ }^{\gamma}\right) \wedge H^{\beta}{ }_{\gamma}+\frac{1}{2}\left(e_{\alpha}\right\rfloor Q_{\beta \gamma}\right) M^{\beta \gamma}, \\
E^{\alpha}{ }_{\beta} & =-\vartheta^{\alpha} \wedge H_{\beta}-M_{\beta}^{\alpha},
\end{aligned}
$$

where $\left.e_{\alpha}\right\rfloor$ denotes the interior product with the frame.

\section{COLLIDING WAVES IN MAG}

This work, as was stated previously, is concerned with fields interpretable as colliding wave solution. With this goal in mind, we extend the definition of vacuum colliding waves, defined by Ernst et al. [12] to MAG theories.

The set of colliding waves solutions in metric-affine gravity is described by the metric

$$
g=2 g(u, v) d u d v+g_{a b}(u, v) d x^{a} d x^{b}, \quad a, b=1,2,
$$

which only depends on the advanced and retarded time $u:=t-z$ and $v:=t+z$, respectively. The domain of the coordinate charts consists of $(x, y) \in \mathbf{R}^{2}$ and $(u, v) \in \mathbf{R}^{2}$; it is the union of four continuous regions: I $:=\{(u, v): 0 \leq u<1,0 \leq v<1\}$, II $:=\{(u, v): u<0,0 \leq$ $v<1\}$, III $:=\{(u, v): 0 \leq u<1, v<0\}$, IV $:=\{(u, v): u \leq 0, v \leq 0\}$, see Fig.1.

As for the torsion and nonmetricity field configurations, we concentrate on the simplest non-trivial case with shear. According to its irreducible decomposition (see the Appendix B of [5]), the nonmetricity contains two covector pieces, namely ${ }^{(4)} Q_{\alpha \beta}=Q g_{\alpha \beta}$, the dilation piece, and

$$
\left.\left.{ }^{(3)} Q_{\alpha \beta}=\frac{4}{9}\left(\vartheta_{(\alpha} e_{\beta}\right\rfloor \Lambda-\frac{1}{4} g_{\alpha \beta} \Lambda\right), \quad \text { with } \quad \Lambda:=\vartheta^{\alpha} e^{\beta}\right\rfloor \ell_{\alpha \beta},
$$

a proper shear piece. Accordingly, our ansatz for the nonmetricity reads

$$
Q_{\alpha \beta}={ }^{(3)} Q_{\alpha \beta}+{ }^{(4)} Q_{\alpha \beta}
$$

The torsion, in addition to its tensor piece, encompasses a covector and an axial covector piece. Let us choose only the covector piece as non-vanishing: 


$$
\left.T^{\alpha}={ }^{(2)} T^{\alpha}=\frac{1}{3} \vartheta^{\alpha} \wedge T, \quad \text { with } \quad T:=e_{\alpha}\right\rfloor T^{\alpha}
$$

Thus we are left with the three non-trivial one-forms $Q, \Lambda$, and $T$. We shall assume that this triplet of one-forms share the spacetime symmetries, i.e. they depend on the variables $u$ and $v$ only. The metric and the triplet fields have to be continuous over the whole domain.

In the region IV, a subregion of the Minkowski space, it is required that

$$
g_{\mu \nu}(u, v)=g_{\mu \nu}(0,0), \quad Q=Q_{0}, \quad \Lambda=\Lambda_{0}, \quad T=T_{0},
$$

which by scale transformations can be brought to standard Minkowski metric and vanishing constants. In region II, the metric components and the triplet of one-forms depends only on $v$, i.e. $g_{\mu \nu}=g_{\mu \nu}(0, v), Q=(0, v), \Lambda=\Lambda(0, v)$, and $T=T(0, v)$. In region III these fields are functions of the coordinate $u$, i.e. $g_{\mu \nu}=g_{\mu \nu}(u, 0), Q=(u, 0), \Lambda=\Lambda(u, 0)$, and $T=T(u, 0)$. In region I, which is occupied by the scattered null fields, the metric components and the triplet are functions of both $u$ and $v$ coordinates.

The metric, the torsion and the nonmetricity fields in region II and III depend only on one variable, i.e. $u$ and $v$, respectively. Each of these regions is equipped with five Killing vectors related with the metric. Moreover, the conformal Weyl tensor part corresponding to the Riemannian part possesses a quadrupole null eigendirection being covariantly constant. These two properties are characteristic of ppN waves. In region II, we have a pp wave, depending only on $v$, propagating to the right, while in region III the pp wave, depending on $u$, propagates to the left. Both waves collide at the event $u=v=0$, and from this event arises the interaction region I. In our case the torsion and nonmetricity depend in the various regions considered in the same way on $u$ and $v$ as the metric. Therefore, our situation describe also torsional and nonmetricity waves which propagate along null directions in regions II and III and collide in region I.

The following ansatz turns to be compatible with the above considerations,

$$
Q=k_{0} \rho(u, v) \vartheta^{\hat{2}}=\frac{k_{0}}{k_{1}} \Lambda=\frac{k_{0}}{k_{2}} T .
$$


Here we introduced a second function $\rho(u, v)$ which has to be determined by the field equations of MAG.

If we take the trace of the zeroth Bianchi identity

$$
D Q_{\alpha \beta}=2 Z_{\alpha \beta}
$$

it merely consists of one irreducible piece $2 d Q=Z_{\gamma}{ }^{\gamma}={ }^{(4)} Z_{\gamma}{ }^{\gamma}$. Consequently, $Q$ serves as a potential for ${ }^{(4)} Z_{\gamma}{ }^{\gamma}$ in the same way as $A$ for $F=d A$. In addition, the third part of (3.7) reads ${ }^{(3)}\left(D Q_{\alpha \beta}\right)=2^{(3)} Z_{\alpha \beta}$, where

$$
\left.\left.{ }^{(3)} Z_{\alpha \beta}=\frac{2}{3}\left(\vartheta_{(\alpha} \wedge e_{\beta)}\right\rfloor \delta-\frac{1}{2} g_{\alpha \beta} \delta\right), \quad \text { with } \quad \delta:=\frac{1}{2} \vartheta^{\alpha} \wedge e^{\beta}\right\rfloor Z_{\alpha \beta} .
$$

The similarity in structure of (3.2) and (3.8) is apparent. Indeed, provided torsion carries only a covector piece, see (3.4), we find

$$
\delta=\frac{1}{6} d \Lambda
$$

i.e. ${ }^{(3)} Q_{\alpha \beta}$ acts as a potential for ${ }^{(3)} Z_{\alpha \beta}$.

In this way, the problem is reduced to know the metric (coframe) and the fuction $\rho$. Thus, the most general form of our fields compatible with colliding wave spacetime structure is given by [13,14]:

$$
\begin{aligned}
& \rho=\rho(u, v), C_{a b c d}^{\star}=2 \Psi_{0} U_{a b} U_{c d}+2 \Psi_{2}\left(U_{a b} V_{c d}+V_{a b} U_{c d}+W_{a b} W_{c d}\right)+2 \Psi_{4} V_{a b} V_{c d}, \quad \text { region I, } \\
& \rho=\rho(v), \quad C_{a b c d}^{\star}=2 \Psi_{0} U_{a b} U_{c d}, \quad \text { region II }, \\
& \rho=\rho(u), \quad C_{a b c d}^{\star}=2 \Psi_{4} V_{a b} V_{c d}, \quad \text { region III },
\end{aligned}
$$

where $C_{a b c d}^{\star}$ is the conformal Weyl tensor corresponding to the Riemannian part of the curvature tensor, and with

$$
\begin{aligned}
W_{a b} & =m_{a} \tilde{m}_{b}-m_{b} \tilde{m}_{a}-k_{a} l_{b}+k_{b} l_{a}, \\
V_{a b} & =k_{a} m_{b}-k_{b} m_{a}, \\
U_{a b} & =-l_{a} \tilde{m}_{b}+l_{b} \tilde{m}_{a} .
\end{aligned}
$$

where $m_{a}, \tilde{m}_{b}, k_{a}$ and $l_{a}$ are null tetrads. In the next section we present an example of these kind of exact solutions. 


\section{COLLIDING WAVE SOLUTION IN MAG}

Let us consider a MAG solution in the interaction region I, i.e. the region arising after the collision of the waves. The coframe in the coordinates $(u, v, x, y)$ reads:

$$
\begin{aligned}
\vartheta^{\hat{0}} & =\sqrt{\Sigma}\left(\frac{d u}{U}-\frac{d v}{V}\right) \\
\vartheta^{\hat{1}} & =\sqrt{\Sigma}\left(\frac{d u}{U}+\frac{d v}{V}\right) \\
\vartheta^{\hat{2}} & =\sqrt{\frac{\Delta}{\Sigma}}\left(d x+j^{2}(u v-U V)^{2} d y\right) \\
\vartheta^{\hat{3}} & =\sqrt{\frac{1}{\Sigma}}(u v-U V)\left\{j d x+\left([\kappa m+\widetilde{a}(u V-v U)]^{2}+j^{2}\right) d y\right\},
\end{aligned}
$$

with two unknown functions $\Sigma(u, v), \Delta(u, v)$. Consequently, the metric is given by

$$
\begin{aligned}
g_{(\mathrm{I})}= & 4 \Sigma \frac{d u}{U} \frac{d v}{V}+\frac{1}{\Sigma}\left\{(u v-U V)^{2}\left[j d x+\left(j^{2}+[\kappa m+\widetilde{a}(u V-v U)]^{2}\right) d y\right]^{2}\right. \\
& \left.+\Delta\left[d x+j^{2}(u v-U V)^{2} d y\right]^{2}\right\}
\end{aligned}
$$

where

$$
\begin{aligned}
U & :=\sqrt{1-u^{2}}, \quad V:=\sqrt{1-v^{2}} \\
\Sigma & =[\widetilde{a}(u V-v U)+\kappa m]^{2}+j^{2}(u V+v U)^{2}, \\
\widetilde{a}^{2} & =m^{2} \kappa^{2}-j^{2}-q_{1}^{2}, \\
\Delta & =\widetilde{a}^{2}(u v+U V)^{2}
\end{aligned}
$$

The nonmetricity and the torsion read as follows:

$$
\begin{aligned}
Q_{(\mathrm{I})}^{\alpha \beta} & \left.=\frac{\widetilde{a}(u V-v U)+\kappa m}{\sqrt{\Sigma \Delta}}\left[k_{0} N o^{\alpha \beta}+\frac{4}{9} k_{1} N\left(\vartheta^{(\alpha} e^{\beta)}\right\rfloor-\frac{1}{4} o^{\alpha \beta}\right)\right] \vartheta^{\hat{2}}, \\
T_{(\mathrm{I})}^{\alpha} & =\frac{k_{2} N}{3} \frac{\widetilde{a}(u V-v U)+\kappa m}{\sqrt{\Sigma \Delta}} \vartheta^{\alpha} \wedge \vartheta^{\hat{2}} .
\end{aligned}
$$

Here $j, m, q_{1}$ and $N$ are arbitrary integration constants, and the coefficients $k_{0}, k_{1}, k_{2}$ in the ansatz (3.6) are determined by the dimensionless coupling constants of the Lagrangian: 


$$
\begin{aligned}
& k_{0}=\left(\frac{a_{2}}{2}-a_{0}\right)\left(8 b_{3}+a_{0}\right)-3\left(c_{3}+a_{0}\right)^{2}, \\
& k_{1}=-9\left[a_{0}\left(\frac{a_{2}}{2}-a_{0}\right)+\left(c_{3}+a_{0}\right)\left(c_{4}+a_{0}\right)\right], \\
& k_{2}=\frac{3}{2}\left[3 a_{0}\left(c_{3}+a_{0}\right)+\left(8 b_{3}+a_{0}\right)\left(c_{4}+a_{0}\right)\right] .
\end{aligned}
$$

A rather weak condition, which must be imposed on these coefficients, prescribes a value for the coupling constant $b_{4}$, namely

$$
b_{4}=\frac{a_{0} k+2 c_{4} k_{2}}{8 k_{0}}, \quad \text { with } \quad k:=3 k_{0}-k_{1}+2 k_{2} .
$$

and the following relation for $z_{4}$

$$
q_{1}^{2}=\kappa z_{4} \frac{\left(k_{0} N\right)^{2}}{2 a_{0}} .
$$

Our solution can be extended to the full spacetime by introducing the Heaviside step function

$$
\Theta(u)=\left\{\begin{array}{ll}
1, & u \geq 0 \\
0, & u<0
\end{array},\right.
$$

with $\Theta^{2}(u)=\Theta(u)$, and replacing $U \rightarrow \sqrt{1-\Theta(u) u^{2}}$ and $V \rightarrow \sqrt{1-\Theta(v) v^{2}}$, cf. [15].

Then in region II the coframe reduces to

$$
\begin{aligned}
\vartheta^{\hat{0}} & =\sqrt{\Sigma}\left(d u-\frac{d v}{V}\right) \\
\vartheta^{\hat{1}} & =\sqrt{\Sigma}\left(d u+\frac{d v}{V}\right) \\
\vartheta^{\hat{2}} & =\sqrt{\frac{\Delta}{\Sigma}}\left(d x+j^{2}\left(1-v^{2}\right) d y\right) \\
\vartheta^{\hat{3}} & =-\sqrt{\frac{1}{\Sigma}} \sqrt{1-v^{2}}\left\{j d x+\left[(\kappa m-\widetilde{a} v)^{2}+j^{2}\right] d y\right\},
\end{aligned}
$$

and the corresponding metric is given by

$$
\begin{aligned}
g_{(\mathrm{II})}= & 4 \Sigma \frac{d u d v}{\sqrt{1-v^{2}}}+\frac{1}{\Sigma}\left(1-v^{2}\right)\left\{\left[j d x+\left[j^{2}+(\kappa m-\widetilde{a} v)^{2}\right] d y\right]^{2}\right. \\
& \left.+\widetilde{a}^{2}\left[d x+j^{2}\left(1-v^{2}\right) d y\right]^{2}\right\}
\end{aligned}
$$

where 


$$
\Sigma=(\kappa m-\widetilde{a} v)^{2}+j^{2} v^{2}, \quad \Delta=\widetilde{a}^{2}\left(1-v^{2}\right)
$$

which represents a plane wave solution (in the sense of Petrov classification, a type N solution [14,[15]). The nonmetricity and the torsion in this region can be written as follows:

$$
\begin{aligned}
Q_{(\mathrm{II})}^{\alpha \beta} & \left.=\frac{\kappa m-\widetilde{a} v}{\sqrt{\Sigma \Delta}}\left[k_{0} N o^{\alpha \beta}+\frac{4}{9} k_{1} N\left(\vartheta^{(\alpha} e^{\beta)}\right\rfloor-\frac{1}{4} o^{\alpha \beta}\right)\right] \vartheta^{\hat{2}} \\
T_{(\mathrm{II})}^{\alpha} & =\frac{k_{2} N}{3} \frac{\kappa m-\widetilde{a} v}{\sqrt{\Sigma \Delta}} \vartheta^{\alpha} \wedge \vartheta^{\hat{2}} .
\end{aligned}
$$

In region III we arrive at the coframe

$$
\begin{aligned}
& \vartheta^{\hat{0}} \quad \sqrt{\Sigma}\left(\frac{d u}{U}-d v\right) \\
& \vartheta^{\hat{1}}=\sqrt{\Sigma}\left(\frac{d u}{U}+d v\right) \\
& \vartheta^{\hat{2}}=\sqrt{\frac{\Delta}{\Sigma}}\left(d x+j^{2}\left(1-u^{2}\right) d y\right) \\
& \vartheta^{\hat{3}}=-\sqrt{\frac{1}{\Sigma}} \sqrt{1-u^{2}}\left\{j d x+\left[(\kappa m+\widetilde{a} u)^{2}+j^{2}\right] d y\right\},
\end{aligned}
$$

and the metric takes the following form:

$$
\begin{aligned}
g_{(\mathrm{III})}= & 4 \Sigma \frac{d u d v}{\sqrt{1-u^{2}}}+\frac{1}{\Sigma}\left(1-u^{2}\right)\left\{\left[j d x+\left[j^{2}+(\kappa m+\widetilde{a} u)^{2}\right] d y\right]^{2}\right. \\
& \left.+\widetilde{a}^{2}\left[d x+j^{2}\left(1-u^{2}\right) d y\right]^{2}\right\}
\end{aligned}
$$

where

$$
\Sigma=(\kappa m+\widetilde{a} u)^{2}+j^{2} u^{2}, \quad \Delta=\widetilde{a}^{2}\left(1-u^{2}\right) .
$$

The nonmetricity and the torsion are now given by

$$
\begin{aligned}
Q_{(\mathrm{III})}^{\alpha \beta} & \left.=\frac{\kappa m+\widetilde{a} u}{\sqrt{\Sigma \Delta}}\left[k_{0} N o^{\alpha \beta}+\frac{4}{9} k_{1} N\left(\vartheta^{(\alpha} e^{\beta)}\right\rfloor-\frac{1}{4} o^{\alpha \beta}\right)\right] \vartheta^{\hat{2}}, \\
T_{(\mathrm{III})}^{\alpha} & =\frac{k_{2} N}{3} \frac{\kappa m+\widetilde{a} u}{\sqrt{\Sigma \Delta}} \vartheta^{\alpha} \wedge \vartheta^{\hat{2}} .
\end{aligned}
$$

Here and in region II, $k_{0}, k_{1}$, and $k_{2}$ still satisfy (4.6), (4.7) and (4.8). It is easy to see that this is also a wave solution. 
Finally, in the flat region IV

$$
\begin{aligned}
\vartheta^{\hat{0}} & =\sqrt{\Sigma}(d u-d v) \\
\vartheta^{\hat{1}} & =\sqrt{\Sigma}(d u+d v) \\
\vartheta^{\hat{2}} & =\sqrt{\frac{\Delta}{\Sigma}}\left(d x+j^{2} d y\right) \\
\vartheta^{\hat{3}} & =-\sqrt{\frac{1}{\Sigma}}\left\{j d x+\left[(k m)^{2}+j^{2}\right] d y\right\} \\
g_{(\mathrm{IV})} & =4 \kappa^{2} m^{2} d u d v+\frac{1}{(\kappa m)^{2}}\left\{\left[j d x+\left[j^{2}+(\kappa m)^{2}\right] d y\right]^{2}+\widetilde{a}^{2}\left[d x+j^{2} d y\right]^{2}\right\},
\end{aligned}
$$

which is always reducible to the flat Minkowski form.

This solution was checked with Reduce [16] with its Excalc package [17 for treating

exterior differential forms [18] and the Reduce-based GRG computer algebra system [19]. The way of derivation of this solution is related to the search of a class of cylindrically symetric solutions in MAG, starting with the line element

$$
d s^{2}=\Delta\left(\frac{d p^{2}}{P(p)}-\frac{d q^{2}}{Q(q)}\right)+\frac{Q}{\Delta}(d \tau+\widetilde{N}(q) d \sigma)^{2}+\frac{P}{\Delta}(d \tau+\widetilde{M}(p) d \sigma)^{2}
$$

with $\Delta:=\widetilde{M}-\widetilde{N}$. Assuming first that $P$ and $Q$ are polynomials up to fourth degree on $p$ and $q$, respectively, second $\widetilde{M}$ and $\widetilde{N}$ are polynomials up to second degree also on $p$ and $q$, and third the torsion and nonmetricity are proportional rational functions, then one arrives at algebraic equations, solvable by computer algebra programs, for the polynomials coefficients. It is always possible to introduce the $u$ and $v$ coordinates through $p=u V+v V$, $q=u V-v U, U=\sqrt{1-u^{2}}$, and $V=\sqrt{1-v^{2}}$. However, only certain solutions satisfy the requirement of Ernst colliding waves (compare ref. 12 and section 3).

\section{DISCUSSION}

As it has been pointed out, the solution presented describes the scattering of two noncollinear polarized gravitation plane waves. At the leading edge of each colliding type-N gravitational wave, the curvature tensor exhibits a jump discontinuity arising, for example, from the second derivative $\left(-U^{2}\right)^{\prime \prime}=u^{2} \delta^{\prime}(u)+4 u \delta(u)+2 \Theta(u)$. The former is interpreted 
as a gravitational impulsive wave, whereas the latter is attributed to a gravitational shock wave.

As far as the nonmetricity and torsion are concerned, if they are considered as fundamental quantities then they behave as continuous functions when crossing different regions; if they were considered as secondary quantities defined by means of derivatives of more fundamental functions, then they could present delta singularities and jump discontinuities. However, even then the Bianchi identities hold in a distributional sense, see [15]. In particular, also $D T^{\alpha}=R_{\beta}^{\alpha} \wedge \vartheta^{\beta}$ holds. There are no problems on the right-hand side because the delta type singularities of the curvature are multiplied by the smooth distributions $\sqrt{1-\Theta(u) u^{2}}$ and $\sqrt{1-\Theta(v) v^{2}}$, respectively.

So far it is not quite clear, if this special MAG model has problems with redundant variables. In the case of restricted Poincaré gauge models (without nonmetricity), a similar reduction (induced via a double duality ansatz) was based on the teleparallelism equivalence, see Baekler et al. Ref. [23]. However, it was shown by Lenzen [22], and later confirmed in Ref. 24] that then necessarily free functions occur in exact torsion solutions. (The tentavive gauge fixing approach suggested there as a way out met considerable criticism.) Thus for the so-called "viable" set there exist infinite many exact vacuum solutions which may indicate a physically problematic degeneracy of those models. Recent reports to rescue the initial value problem in PG theory by Hecht et al. [21] and the Refs. therein, seem not to be conclusive.

The related situation for MAG is not yet resolved, since again a teleparallelism type relation, see (5.9.16) of Ref. [5], seems to be crucial for the equivalence proof of MAG with the Einstein-Proca Lagrangian. Already earlier, within the framework of the Poincaré gauge theory $(\mathrm{PG})$ of gravity, the post-Newtonian generation of gravitational radiation in one parameter teleparallelism type $T^{2}$ models were studied by Schweizer et al. 20. In a first order approximation no deviation from Einstein's GR was found; also, as a bonus, the dipole gravitational radiation of other alternative theories is absent here.

More recently, plane wave solutions of GR are generalized to $R+R^{2}+T^{2}$ models by Zhytnikov [26]. (Note here the possibility of notational confusion, since $Q$ is there used for 
torsion.) Our present paper is an extension of this work in two different directions: First we extend to models with nonmetricity $Q_{\alpha \beta}$ including the Weyl covector $Q$ and, secondly, also colliding waves exhibiting shock fronts are considered.

\section{ACKNOWLEDGMENTS}

We would like to thank Friedrich W. Hehl for useful discussions and literature hints. This work was partially supported by CONACyT, grants No. 3544-E9311, No. 3898P-E9608, No. 3692P-E9607 and by the joint German-Mexican project KFA-Conacyt E130-2924 and DLR-Conacyt 6.B0a.6A. Moreover, E.W.M. acknowledges the support by the short-term fellowship 9616160156 of the German Academic Exchange Service (DAAD), Bonn, and C.L. thanks the Deutsche Forschungsgemeinschaft and the DAAD for financial support. 


\section{REFERENCES}

[1] K. Khan and R. Penrose, Nature 229 (1971) 185.

[2] P. Szekeres, J. Math. Phys. 13 (1972) 286.

[3] S. Chandrasekhar and V. Ferrari, Proc. R. Soc. London Ser. A396 (1984) 55.

[4] S. Chandrasekhar and B.C. Xanthopoulos, Proc. R. Soc. London Ser. A398 (1985) 223.

[5] F.W. Hehl, J.D. McCrea, E.W. Mielke, and Y. Ne'eman, Phys. Rep. 258 (1995) 1.

[6] R. Tresguerres, Z. Phys. C65 (1995) 347.

[7] R. Tresguerres, Phys. Lett. A200 (1995) 405.

[8] R.W. Tucker and C. Wang, Class. Quantum Grav. 12 (1995) 2587.

[9] T. Dereli, M. Önder, J. Schray, R.W. Tucker, and C. Wang, Class. Quantum Grav. 13 (1996) L103.

[10] A. Macías, E.W. Mielke and J. Socorro, Class. Quantum Grav. (1997). In print.

[11] J. Socorro, C. Lämmerzahl, A. Macías, and E.W. Mielke: "Multipole solutions in metric-affine gravity". (1997) Submitted.

[12] F.J. Ernst, A. García, and I. Hauser, J. Math. Phys. 28 (1987) 2951.

[13] A. García and N. Bretón, Phys. Rev. D53 (1996) 4351.

[14] A. García, J. Math. Phys. 25 (1984) 1951.

[15] A.H. Taub, J. Math. Phys. 21 (1980) 1423.

[16] A.C. Hearn, REDUCE User's Manual. Version 3.6. Rand publication CP78 (Rev. 7/95) (RAND, Santa Monica, CA 90407-2138, USA, 1995).

[17] E. Schrüfer, F.W. Hehl, and J.D. McCrea, Gen. Relat. Grav. 19 (1987) 197. 
[18] D. Stauffer, F.W. Hehl, N. Ito, V. Winkelmann, and J.G. Zabolitzky: Computer Simulation and Computer Algebra - Lectures for Beginners. 3rd ed. (Springer, Berlin, 1993).

[19] V.V. Zhytnikov, GRG. Computer Algebra System for Differential Geometry, Gravity and Field Theory. Version 3.1 (Moscow, 1991) 108 pages.

[20] M. Schweitzer, N. Straumann, and A. Wipf, Gen. Rel. Grav. 12 (1980) 951.

[21] R.D. Hecht, J.M. Nester, and V.V. Zhytnikov, Phys. Lett. A222 (1996) 37.

[22] J. Lenzen, Nuovo Cim. B82 (1984) 85.

[23] P. Baekler and E.W. Mielke, Phys. Letters 113A (1986) 471.

[24] P. Baekler and E.W. Mielke, Fortschr. Phys. 36 (1988) 549.

[25] M. Kaku: Quantum Field Theory (Oxford University Press 1993). p. 750.

[26] V.V. Zhytnikov, J. Math. Phys. 35 (1994) 6001. 


\section{FIGURES}

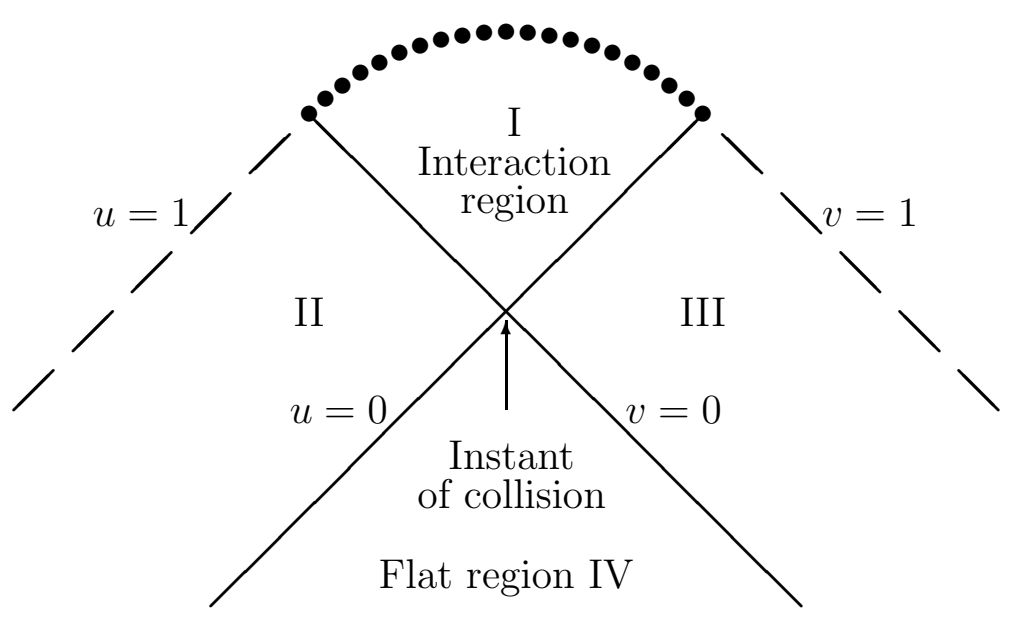

FIG. 1. The four regions of the spacetime: Region IV where the waves propagate is flat. The impulsive gravitational waves propagate along the null boundaries $v=0$ and $u=0$, separating regions II and IV, and III and IV, respectively. In region II, observers see the shower of pure gravitational radiation following the wave front propagating along $v=0$. Symmetrical consideration apply in region III. The collision occurs at $(0,0)$ and the interaction is described by region I. 